\title{
Reduction of surgical site infection using a microbial sealant: A randomized trial
}

\author{
Anand Iyer, MCh, Ian Gilfillan, FRACS, Sanjay Thakur, MCh, and Sanjay Sharma, FRACS
}

Objective: Surgical site infection is a major cause of mortality and morbidity. We have explored the use of a mi-
crobial sealant applied before the surgical incision to reduce surgical site infection.

\begin{abstract}
Methods: We conducted a prospective, randomized, controlled clinical trial to determine the efficacy of a cyanoacrylate sealant in patients undergoing coronary artery bypass grafting. Both left and right long saphenous veins were harvested in individual patients below the knee if 3 or more lengths of vein were required. The sealant (Kimberly-Clark InteguSeal, Roswell, Ga) was applied to 1 leg chosen randomly, and the other leg was prepared in a conventional fashion. Microbiological swabs from the leg wounds were taken at 5 days, and wounds were assessed according to the Southampton score at 30 days by 2 blinded observers.
\end{abstract}

Results: The baseline characteristics of the treated and untreated legs were similar because the procedure was conducted on each individual patient. The study was terminated at 47 patients after review. Patients in whom the sealant was used had $1(2.1 \%)$ wound infection, and there were $12(25.5 \%)$ wound infections in the conventionally prepared $\operatorname{leg}(P=.001)$. There were 13 positive cultures from the treated leg and 22 positive cultures from the untreated site.

Conclusions: The microbial skin sealant applied immediately before the incision significantly reduced the rate of surgical site infection. There was no sensitivity or adverse reaction after application. The treatment was easily integrated with existing routine preoperative procedures. Microbial sealant may thus be a useful addition to a multimodal approach to minimize surgical site infection. (J Thorac Cardiovasc Surg 2011;142:438-42)

The human body contains $10^{13}$ human cells, but also has $10^{14}$ resident bacteria. Consequently, any breach in the skin creates a route for these bacteria to enter the body and potentially create an infection. ${ }^{1,2}$ Infection risk is directly proportional to the dose of bacterial contamination and the virulence of the organism but inversely proportional to the resistance of the host. Potential sources of contamination include the surgical personnel, breaks in sterile technique, operating room environment, and materials carried into the operating room. However, a significant alternative source of pathogens is from the patient's own skin flora, mucous membranes, and hollow viscera.

Data from the US Centers for Disease Control National Nosocomial Infections Surveillance system show that surgical site infections (SSIs) are the third most frequently reported nosocomial infections, accounting for $14 \%$ to $16 \%$ of such infections among hospitalized patients and $38 \%$ among surgical patients. ${ }^{3,4}$ SSIs impose a substantial clinical burden.

From the Department of Cardiothoracic Surgery, Fremantle Hospital, Fremantle, Western Australia, Australia.

Registered with the Australian New Zealand Clinical Trials Registry: ACTRN 126100007.

Disclosures: Authors have nothing to disclose with regard to commercial support.

Received for publication Nov 26, 2010; revisions received Jan 12, 2011; accepted for publication Feb 9, 2011; available ahead of print March 28, 2011.

Address for reprints: Anand Iyer, MCh, Department of Cardiothoracic Surgery, Fremantle Hospital, Alma Street, Fremantle, Western Australia: 6160, Australia (E-mail: anandcvts@hotmail.com).

0022-5223/\$36.00

Crown Copyright (C 2011 Published by Elsevier Inc. on behalf of The American Association for Thoracic Surgery

doi:10.1016/j.jtcvs.2011.02.014
Patients with SSIs are more likely to require readmission to hospital or intensive care unit treatment and are at higher risk of death than those without such infections. In a casecontrolled study involving 215 matched pairs of patients with and without SSIs, the relative risk was 2.2 (95\% confidence interval $[\mathrm{CI}], 1.1-4.5)$ for death associated with SSIs, 5.5 (CI, 4.0-7.7) for readmission, and $1.6(\mathrm{CI}, 1.3-2.0)^{5}$ for intensive care unit treatment. Moreover, patients with SSIs required longer hospitalization; the median duration of hospitalization in infected patients was 11 days, compared with 6 days in uninfected patients. Similarly, a review of the incidence and health economic implications of SSIs in Europe found that the mean length of extended hospitalization was 9.8 days. $^{6}$ Consequently, SSIs are responsible for a considerable burden on healthcare costs. In the case-control study described, ${ }^{5}$ the median excess cost associated with SSIs during a first hospitalization was \$3089 (95\% CI, \$2139-4163), and this figure increased to $\$ 5038$ in patients who required readmission. ${ }^{5}$ Similarly, European data suggest that the mean cost of prolonged hospitalization due to SSIs is $€ 325$ per day. ${ }^{6}$ Deep SSIs involving internal organs or body cavities are associated with even longer hospital stays and further increases in costs, compared with SSIs that affect only the incision. ${ }^{3}$

Postoperative SSI is a particularly serious complication in patients undergoing cardiac surgery. Infected wounds usually require prolonged therapy and hospitalization. Sternal wound complications result in increased morbidity and mortality, reaching $10 \%$ to $40 \%{ }^{7-9}$

Efforts to reduce the rate of SSI in all types of surgery have been directed at modifying risk factors. Procedures focused 


\section{Abbreviations and Acronyms \\ $\mathrm{CABG}=$ coronary artery bypass graft \\ $\mathrm{CI}=$ confidence interval \\ SSI $=$ surgical site infection}

on reducing bacterial contamination of the surgical site, administering appropriate antibiotic prophylaxis, and using meticulous surgical technique. ${ }^{10}$ Scrubbing of the operative site with a variety of antiseptic solutions before surgery has been studied, and although this substantially reduces the skin flora, approximately $20 \%$ of the microbes remain buried deep in hair follicles and sweat glands and persist after scrubbing. ${ }^{11}$ Thus, complete sterilization of the skin is not possible. The use of a microbial sealant in the preparation of a patient for surgery is a recent technologic development with the potential to reduce rates of SSI. The sealant forms a breathable barrier that prevents migration of skin flora into the incision. In contrast with conventional skin preparations, the sealant also seals microabrasions on the skin, thus preventing recolonization of such spaces with residual potential pathogens after skin sterilization. Because the sealant physically locks down the pathogens, there is no potential for the development of microbial antibiotic resistance. This article reports the effect of pretreatment with n-butyl cyanoacrylate-based microbial skin sealant in a population undergoing cardiac surgery and discusses its potential use in decreasing infections in other kinds of surgical procedures.

\section{MATERIALS AND METHODS Study Population}

We began the trial in August 2008 after the research was approved by the institutional ethics committee registered with the Australian New Zealand Clinical Trials Registry. Forty-seven patients were studied; recruitment was halted at this number after further ethical committee review. Patient follow-up was complete at 1 month. Patients were eligible for inclusion in the study if undergoing coronary artery bypass graft (CABG) surgery and requiring 3 or more lengths of long saphenous vein to achieve revascularization. Additional internal thoracic conduit was also routinely used. The long saphenous vein grafts were taken from the below-knee segment in both legs of all patients. The sealant was applied to only 1 leg per case, selected randomly. The other leg thus acted as a matched control. The sealant was not used on the sternal incision. All patients gave written informed consent. Major exclusion criteria were if the patient required $\leq 2$ segments of vein wherein only 1 leg would be used and any features that would cause dissimilarity between the legs, which included the vein in either leg not being usable, unilateral vascular disease, or skin lesion. Patients were also excluded if there was a discrepancy in length of the skin incision between the treated and untreated legs. By comparing 1 leg with the other, any bias with regard to patient characteristics was avoided. Patient characteristics are shown in Table 1.

\section{Surgical Technique}

Apart from the application of the sealant to the treated leg, surgical techniques for both legs were identical. Hair removal was performed using an electrical clipper the day before surgery. Patients then washed by soap on the morning of surgery. The skin was disinfected using alcoholic povidoneiodine solution, and 3 minutes were allowed for the disinfecting solution to dry before the application of microbial sealant. The microbial sealant was applied to one of the legs on a random basis. No sham application was used in the untreated leg. The InteguSeal IS 100 (Kimberly-Clark, Roswell, Ga) applicator was used to apply a single, even layer of microbial sealant over an area overlying the saphenous vein harvest site. The cost of 1 applicator was $\mathrm{A} \$ 30$, which was paid by the hospital. Another delay of 2 minutes allowed the sealant to dry. Iodine-impregnated drapes were used in all patients (Ioban; 3M, St Paul, Minn). Veins were harvested by a single open incision. Endoscopic techniques were not adopted, and in most of the patients the below portion of veins was harvested. Standard surgical procedure involved closure of subcutaneous layer with 2-0 Vicryl (Covidien, Mansfield, Mass/US Surgical, Norwalk, Conn) and subcuticular closure of the skin with 3-0 Caprosyn (Covidien/US Surgical). A hydrocolloid dressing, Comfeel (Coloplast, Humlebaek, Denmark) was used in all patients. All patients underwent cardiopulmonary bypass with systemic cooling to $32^{\circ} \mathrm{C}$ to $34^{\circ} \mathrm{C}$. All patients underwent standard bypass grafts. None of the patients in the study had perioperative infarctions, and there were no adverse intraoperative events. There was no correlation of infection with the bypass time. The incisions were approximately the same length in both legs in most of the patients, but this had no bearing in causing infections.

\section{Study Outcomes}

The wounds of all the patients were examined daily during the postoperative stay, and a wound swab was taken from both legs on the fourth postoperative day. If there was infection, the swabs were taken from that particular region. If there was no infection, a culture swab was applied to a segment of skin incision. The bacterial isolates obtained from the wounds of all patients along the incision site before their discharge were identified using routine microbiological diagnostic procedures. Preoperative swabs were not taken. If the wound was showing signs of infection, it was graded per the Southampton wound grading system. ${ }^{12}$ Findings were recorded, and the wound infection was managed accordingly. All patients were followed up by 1 of 2 blinded observers (who were registrars in the unit) at 4 weeks postdischarge when the wounds were examined. Findings were recorded. If a general practitioner involved with the care after discharge detected an infection before the 4-week follow-up, an appointment was arranged before the stipulated follow-up and the findings were recorded.

The Southampton score divides wounds into 5 grades: $0=$ normal healing; grade $1=$ normal healing with bruising or erythema, subclassified as A, B, or $\mathrm{C}$ according to the size of bruising; grade $2=$ erythema with other signs of inflammation, subclassified as A, B, or C according to findings at 1 point, around the sutures, or around the whole wound; grade $3=$ haemoserous discharge $(\mathrm{A}<2 \mathrm{~cm}, \mathrm{~B}>2 \mathrm{~cm}, \mathrm{C}=$ large volume, $\mathrm{D}>3$ days); grade $4=$ purulent discharge $(\mathrm{A}<2 \mathrm{~cm}, \mathrm{~B}>2 \mathrm{~cm})$; and grade $5=$ deep or severe infection with or without tissue breakdown. ${ }^{12}$

\section{Statistical Analysis}

The study design was a prospective, randomized, double-blind trial, and variables were analyzed using the $z$ test.

\section{RESULTS}

Forty-seven patients (ie, 94 legs) were enrolled. In the sealant group, 1 leg developed a severe infection and required incision and drainage, and Staphylococcus aureus was isolated. In the same patient, the untreated leg had no infection. In the remaining 46 treated legs, there was no infection observed at 30 days. We are not aware of any infection developing beyond this point. In the 46 uninfected 


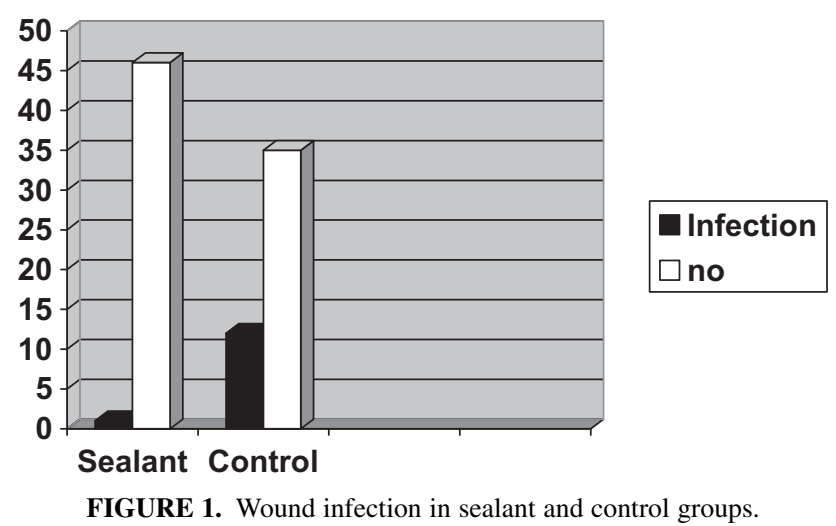

treated legs, $12(26.08 \%)$ grew organisms on culture, the majority of which were skin flora and $S$ epidermidis.

Twelve legs in the untreated group showed evidence of infection that ranged from oozing of serous fluid, which responded to conservative therapy, to severely infected wound requiring debridement (Figure 1). Four patients required incision and drainage, and 1 patient required a debridement. Of the remaining 35 healed legs, $14(40 \%)$ had a positive culture. None of the infected patients had long-term consequences (Table 2).

\section{Statistical Analysis}

Group 1 (sealant) included 47 patients, and group 2 (no sealant) included 47 patients $(P=.0011 ; 95 \% \mathrm{CI}$ for difference, -0.374 to -0.0945$)$. There was a significant difference in the proportions of individuals with infection within the 2 groups.

\section{DISCUSSION}

In cardiac surgery, sternal wound infection continues to be one of the most serious postoperative complications. ${ }^{13}$ Coagulase-negative staphylococci have become the most common causative agents of sternal wound infection, followed by $S$ aureus and others, such as the gram-negative rods. ${ }^{13,14}$ Although the frequency of sternal infection is reported to be low, between $0.7 \%$ and $3.3 \%$, the costs associated with multiple procedures and increased hospital stay should not be underestimated. ${ }^{15,16}$ SSIs in

TABLE 1. Patient characteristics

\begin{tabular}{lc}
\hline Age (y), mean (SD) & $67.0(7.6)$ \\
$\quad$ Male & $39(82.9 \%)$ \\
Female & $8(17.02 \%)$ \\
Hyperlipidemia & $40(85.1 \%)$ \\
Arterial hypertension & $31(65.9 \%)$ \\
Diabetes mellitus & $12(25.5 \%)$ \\
Peripheral vascular disease & $0(0)$ \\
Renal failure & $4(8.5 \%)$ \\
Ejection fraction, mean (SD) & $47.3(13.3)$ \\
Venous disease in the leg & 0 \\
\hline$S D$, Standard deviation. &
\end{tabular}

any specialty have their own implications with regard to patient morbidity and mortality, hospital stay, and cost. It was estimated that there are $175,153(95 \%$ credible interval, 155,911:195,168) cases of healthcare-acquired infection among admissions to Australian hospitals annually, and the extra stay in the hospital to treat symptoms accounts for 854,289 bed days $(95 \%$ credible interval, 645,091: $1,096,244)$. If rates were reduced by $1 \%$, then 150,158 bed days would be released for alternative uses. ${ }^{17}$ On the basis of the findings of a Victorian Nosocomial Infection Surveillance System (VICNISS) study ${ }^{18}$ investigating the impact of hospital-acquired infections on the health system, the 225 SSIs reported between July 2005 and June 2008 in Western Australia are estimated to have cost A $\$ 8.1$ million and consumed 5800 additional bed days. ${ }^{18}$

We performed this trial in leg wounds. Leg wound complications have been reported to occur in $2 \%$ to $24 \%$ of cases $^{19,20}$ and has been as high as $42.8 \%$ in one series. ${ }^{21}$ The incidence of wound infection in the leg has been 12 of 47 cases in the untreated legs because the criteria according to Southampton score is stringent, and even minimal erythema or discharge has been included as infection. We have followed patients for 1 month after discharge and reviewed them before if there were any doubts raised about infection by the general practitioner and even minimal erythema or small discharge in the wounds were considered infections. Some patients were discharged without any issues and during the follow-up were noted to have signs of infection. Although postdischarge follow-up is good in many centers, there is a likelihood that such events may be managed exclusively by the practitioner without referral to the primary unit if not specified in the discharge note. The ability to track events that occur and are managed on an outpatient basis may be less reliable, ${ }^{22}$ and the true incidence of leg infections may be underreported.

The trial wasrandomized without bias because 1 leg in the same patient was compared with the other. We also used the below-knee portion of the vein in most of the patients for grafting because we have encountered increased wound infections in the thigh region. According to Akowuah and colleagues, ${ }^{23}$ the ASEPSIS score (Additional treatment, Serous discharge, Erythema, Purulent exudates, Separation of deep tissues, Isolation of bacteria, Stay in hospital prolonged over 14 days) is reduced when vein harvest is restricted to below the level of the knee. This study found that the use of a microbial sealant before incision significantly reduced the rate of leg wound infections among patients undergoing CABG.

The risk of SSI is considered elevated when the level of contamination is high, although lower doses may be required if foreign material, such as sutures, is present. ${ }^{3}$ In an in vitro skin incision model, recovery of methicillin-resistant $S$ aureus was reduced by $99.9 \%$ with the sealant, whereas the recoveries of $S$ epidermidis and Escherichia coli were 
TABLE 2. Wound infections and cultures in the study population (with and without microbial sealant)

\section{Infection graded per Southampton score}

(isolated organism)

No infection (isolated organism)

Microbial sealant

1. Grade 4B Staphylococcus aureus

34 no growth
4 skin flora
4 coagulase-negative
Staphylococcus
1 Enterococcus
1 Klebsiella
1 coliform
1 Enterobacter (light growth)
22 no growth
9 coagulase-negative
Staphylococcus
2 skin flora
1 Klebsiella
1 coliform

Untreated legs

1. Grade 3D $S$ aureus + coliforms

2. Grade 5 Escherichia coli

3. Grade $5 S$ aureus

4. Grade 4B Klebsiella + E coli

5. Grade 3D skin flora

6. Grade 3B no growth

7. Grade 5 skin flora

8. Grade $3 \mathrm{~A}$ no growth

9. Grade 3B Serratia marcescens

10. Grade 3B $S$ epidermidis

11. Grade 3B $S$ epidermidis

12. Grade 4B Enterobacter aerogenes

reduced by $99.5 \%$ and $96.6 \%$, respectively. ${ }^{24}$ In another study, the microbial sealant reduced the recovery of drug-resistant Acinetobacter baumannii by a factor of $10^{1.5}$. A baumannii is another pathogen that has been implicated in SSIs in patients receiving antimicrobial prophylaxis. ${ }^{5}$

SSIs impose a substantial burden of mortality and morbidity, which increase the demands on resources. This study lends evidence toward implementing sealants into routine clinical practice as being appropriate and supports the findings of Dohmen and colleagues ${ }^{25}$ who showed that sealants significantly reduced sternal wound infection rates.

Cyanoacrylate may act as a skin irritant and cause an allergic skin reaction, but we have found no reports of toxicity or allergies to butyl cyanoacrylate, which is being used as the sealant.

\section{CONCLUSIONS}

The use of a microbial skin sealant before surgery reduced the rate of SSI among patients undergoing CABG. There were no cases of skin sensitivity or other reaction after application of the microbial sealant, which can be used with a variety of skin preparation solutions and most wound closure techniques. The pretreatment is straightforward and has been easily integrated with existing routine preoperative procedures at this surgical center. Microbial sealant may thus be a useful addition to the multimodal approach to minimize bacterial contamination of surgical incisions, which is key in SSI prevention. It is likely that these outcomes can be reproduced in other clean surgical procedures.
The authors thank Leon Neethling (research officer) for performing the statistical analysis of the data.

\section{References}

1. Plowman R, Graves N, Griffin M, Roberts J, Swan T, Cookson B, Taylor L. The Socioeconomic Burden of Hospital Acquired Infection. London: Public Health Laboratory Service; 1999.

2. Plowman R, Graves N, Griffin M, et al. The rate and cost of hospital acquired infections occurring in patients admitted to selected specialties of a district general hospital in England and the national burden imposed. J Hosp Infect. 2001;47: 198-209.

3. Mangram AJ, Horan TC, Pearson ML, Silver LC, Jarvis WR. Guideline for prevention of surgical site infection, 1999: Hospital Infection control Practices Advisory Committee. Infect Control Hosp Epidemiol. 1999;20: 247-78.

4. Emori TG, Gaynes RP. An overview of nosocomial infections, including the role of the microbiology laboratory. Clin Microbiol Rev. 1993;6:428-42.

5. Kirkland KB, Briggs JP, Trivette SL, Wilkinson WE, Sexton DJ. The impact of surgical-site infections in the 1990s: attributable mortality, excess length of hospitalization, and extra costs. Infect Control Hosp Epidemiol. 1999; 20:725-30.

6. DiPiro JT, Martindale RG, Bakst A, Vacani PF, Watson P, Miller MT. Infection in surgical patients: effects on mortality, hospitalization, and postdischarge care. Am J Health Syst Pharm. 1998;55:777-81.

7. Fowler VG Jr, O'Brien SM, Muhlbaier LH, Corey GR, Ferguson TB, Peterson ED. Clinical predictors of major infections after cardiac surgery. Circulation. 2005;112:I358-65.

8. Omran SA, Karimi A, Ahmadi SH, et al. Superficial and deep sternal wound infection after more than 9000 coronary artery bypass graft (CABG): incidence, risk factors and mortality. BMC Infect Dis. 2007;7:112.

9. Shih CC, Shih CM, Su YY, Lin SJ. Potential risk of sternal wires. Eur J Cardiothorac Surg. 2004;25:812-8.

10. Johnston DH, Fairclough JA, Brown EM, Morris R. Rate of bacterial recolonization of the skin after preparation: four methods compared. Br J Surg. 1987; 74:64.

11. Jepsen OB, Bruttomesso KA. The effectiveness of preoperative skin preparations. An integrative review of the literature. AORN J. 1993;58:477-9, 482-4. 
12. Bailey IS, Karran SE, Toyn K, Brough P, Ranaboldo C, Karran SJ. Community surveillance of complications after hernia surgery. Br Med J. 1992;304:469-71.

13. Tegnell A, Aren C, Ohman L. Coagulase-negative staphylococci and sternal infections after cardiac operation. Ann Thorac Surg. 2000;69:1104-9.

14. Stahle E, Tammelin A, Bergstrom R, Hambreus A, Nystrom SO, Hansson HE. Sternal wound complications-incidence, microbiology and risk factors. Eur $J$ Cardiothorac Surg. 1997;11:1146-53.

15. Lepelletier D, Perrron S, Bizouarn P, et al. Surgical site infection after cardiac surgery: incidence, microbiology and risk factors. Infect Control Hosp Epidemiol. 2005;26:466-72.

16. Ridderstolpe L, Gill H, Granfeldt H, et al. Superficial and deep sternal wound complications: incidence, risk factors and mortality. Eur J Cardiothorac Surg. 2001;20:1168-75.

17. Graves N, Halton K, Paterson D, Whitby M. Economic rationale for infection control in Australian hospitals. Healthcare Infection. 2009;14:81-8.

18. VICNISS. VICNISS Hospital Acquired Infection Project, Year 4 report, August 2006. Melbourne: Department of Human Services; 2006. Available at: http:// www.vicniss.org.au/AnnualReport.aspx. Accessed July 2009.
19. Mullen JC, Bentley MJ, Mong K, et al. Reduction of leg wound infections following coronary artery bypass surgery. Can J Cardiol. 1999;15:65-8.

20. Folliguet TA, Le Bret E, Moneta A, et al. Endoscopic vein harvesting versus open technique: a prospective study. Eur J Cardiothorac Surg. 1998;13:662-6.

21. Wipke-Tevis DD, Stotts NA, Skov P, Carrieri-Kohlman V. Frequency, manifestations, and correlates of impaired healing of saphenous vein harvest incisions. Heart Lung. 1996;25:108-16.

22. Keith B. Allen saphenectomy wound complications: the real story. J Thorac Cardiovasc Surg. 2004;127:1535.

23. Akowuah E, Shrivastava V, Ponniah A, Jamnadas B, Chilton G, Cooper G. Above-knee vein harvest for coronary revascularization increases ASEPSIS score. Asian Cardiovasc Thorac Ann. 2006;14:57-9.

24. Wilson SE. Microbial sealing: a new approach to reducing contamination. J Hosp Infect. 2008;70(Suppl 2):11-4.

25. Dohmen PM, Gabbieri D, Weymann A, Linneweber J, Konertz W. Reduction in surgical site infection in patients treated with microbial sealant prior to coronary artery bypass graft surgery: a case-control study. J Hosp Infect. 2009; 72:119-26. 